\title{
Food protein-induced enterocolitis syndrome presenting after necrotizing enterocolitis in a preterm neonate: a case report
}

\author{
Yan Lu, Zhi-Qun Zhang \\ Department of Neonatology, Affiliated Hangzhou First People's Hospital, Zhejiang University School of Medicine, Hangzhou, China \\ Correspondence to: Zhi-Qun Zhang, PhD. Chief Doctor, Department of Neonatology, Affiliated Hangzhou First People's Hospital, Zhejiang \\ University School of Medicine, No. 261 Huansha Road, Hangzhou 310000, China. Email: zhiqun.zhang@zju.edu.cn.
}

\begin{abstract}
When bloody stools occur in a very-low-birth-weight infant in the neonatal intensive care unit (NICU), necrotizing enterocolitis (NEC) is a prime consideration, though food protein-induced enterocolitis syndrome (FPIES) can be causative and is difficult to distinguish from NEC. Food allergy is an adverse reaction following exposure to food due to an abnormal immunologic response to food, and cow's milk allergy (CMA) is the most likely form of food allergy in infants. The clinical features and proper management of patients with FPIES are important to differentiate FPIES from NEC. However, there are very few study reports of preterm infants presenting with food allergy-induced enterocolitis after NEC. Here, we report a case of a very-low-birth-weight infant born at 28 weeks of gestational age who developed recurrent episodes of bloody stools when he was fed cow's milk or given breast milk fortified with milk after NEC recovery on day of life (DOL) 29, 46, and 54. A male preterm infant born at 28 weeks of gestational age presented with bloody stools on DOL 7. He was diagnosed with early-onset NEC with abdominal tenderness, sluggish bowel sounds, increased C-reactive protein (CRP) level and pneumatosis intestinalis (PI). After recovery from NEC on DOL 20, the infant developed three recurrent episodes of bloody stools after being fed cow's milk or breast milk fortified with dairy milk. He was suspected of having recurrent episodes of NEC, but the infant was fairly healthy and did not present abdominal tenderness or abnormal bowel sounds on physical examination. Consecutive blood tests revealed normal CRP levels and increasing eosinophil levels. Abdominal radiograph revealed mild thickening of the small bowel, with no evidence of PI. The infant was finally diagnosed with FPIES in addition to NEC. After the infant received hydrolyzed formula, the bloody stool symptoms were finally resolved. Our case suggests that infants with recurrent episodes of bloody stools with increasing systemic eosinophils count should be considered for the diagnosis of FPIES with cow's milk formula. Rapid improvement and non-progression of systemic symptoms and signs after removing exposure to milk protein may differentiate FPIES from NEC.
\end{abstract}

Keywords: Bloody stool; eosinophilia; food protein-induced enterocolitis syndrome (FPIES); necrotizing enterocolitis (NEC); preterm infant

Submitted Jan 08, 2021. Accepted for publication Mar 12, 2021.

doi: $10.21037 /$ tp-21-9

View this article at: http://dx.doi.org/10.21037/tp-21-9

\section{Introduction}

There are multiple aetiologies of bloody stools in infants. Clinically unstable, premature, or very-low-birth-weight infants should be evaluated for necrotizing enterocolitis (NEC) (1). It is one of the leading causes of morbidity and mortality in preterm infants. The pathophysiology of NEC is inflammation of the intestine leading to bacterial invasion causing necrosis of the colon and the intestine (2). As NEC progresses, it can lead to intestinal perforation causing peritonitis, sepsis, and even death. In the clinical setting, approximately half of all infants with NEC respond to bowel rest, antibiotics, and supportive medical measures, but others develop progressive bowel disease and require surgical 
Table 1 Laboratory tests of the infant with bloody stools

\begin{tabular}{|c|c|c|c|c|}
\hline Variables & $\frac{\text { NEC }}{\text { DOL7 }}$ & \multicolumn{3}{|c|}{ Allergic colitis } \\
\hline WBC $\left(/ \mathrm{cm}^{3}\right) \times 10^{3} / \mu \mathrm{L}$ & 3.3 & 11.8 & 4.2 & 5.1 \\
\hline EOS (\%) & 0.3 & 0.4 & 4.6 & 13.8 \\
\hline CRP (mg/L) & 34 & 2 & 2 & 2 \\
\hline
\end{tabular}

CRP, C-reactive protein; DOL, day of life; EOS, eosinophil; WBC, white blood cell count; CRP, C-reactive protein; FOB, fecal occult blood.

intervention; a third of these patients eventually die (3).

Food protein-induced enterocolitis syndrome (FPIES) is a non-IgE cell-mediated gastrointestinal food allergy that is characterized by inflammatory changes in the distal colon in response to one or more different food proteins (4). The symptoms typically begin in the first month of life. The infants present with bloody, loose stools with mucus. Some can also present with gagging, food refusal and irritability (5). There is no specific laboratory test or radiographic finding for FPIES, but intramural gas has been documented in FPIES (6). FPIES can be misdiagnosed as NEC, which often leads to increased morbidity from recurring episodes, extensive evaluations, and hospitalization before the diagnosis is finally considered $(7,8)$.

We report a case of a very-low-birth-weight infant born at 28 weeks of gestational age with recurrent episodes of bloody stools after feeding with cow's milk or breast milk fortified with milk on day of life (DOL) 29, 46, and 54 after NEC recovery on DOL 20. Complicated by the recurrence of NEC, the diagnosis of the infant was confirmed to be FPIES. Elimination of food allergies ultimately showed resolution of symptoms. We present the following article in accordance with the CARE reporting checklist (available at: http://dx.doi.org/10.21037/tp-21-9).

\section{Case presentation}

A male preterm infant was born to a 28 -year-old gravida 1, para 1 mother at 28 weeks of gestation. The mother received dexamethasone, magnesium sulphate, and antibiotics before labour. The mother's prenatal screening serological tests were all negative. Due to abnormal fetal position with threatened pre-term labour, the infant was delivered through a caesarean section (C-section) with a birth weight of $1,240 \mathrm{~g}$. The amniotic fluid was clear, and the infant had Apgar scores of 7 and 9 at 1 and 5 minutes, respectively. The infant was treated for respiratory distress syndrome at birth with nasal continuous positive airway pressure (nCPAP) for six days, and caffeine citrate was administered for the treatment of apnoea of prematurity on DOL 2. Preterm formula was started on DOL 2 and continued with freshly expressed breast milk (EBM). Feedings were advanced at $10-20 \mathrm{~mL} / \mathrm{kg} / \mathrm{d}$, and the patient tolerated the procedure well.

On DOL 7, the infant developed an increased gastric residual, apnoea, and grossly bloody stools while on $40 \mathrm{~mL} / \mathrm{kg}$.d enteral feeding. Physical examination showed abdominal distention, left abdominal tenderness, and sluggish bowel sounds. Laboratory tests showed increased C-reactive protein (CRP) levels, decreased white blood cell (WBC) levels, and positive stool occult blood (Table 1). An abdominal $\mathrm{X}$-ray demonstrated pneumatosis intestinalis (PI) of the descending colon (Figure 1). Feeds were stopped, and intravenous antibiotics were started. Subsequently, the infant became pale and less active, with episodes of tachycardia and hypotension requiring conventional mechanical ventilation, fluid resuscitation and haemodynamic support for normal blood pressure over the next 24 hours. The infant was managed medically as having definitive NEC (stage 3A) with IV antibiotics (piperacillin/ tazobactam and vancomycin). Two days later, blood culture was positive for Escherichia coli (E. coli), so vancomycin was stopped, and the antibiotics were changed to a combination of piperacillin/tazobactam and meropenem for 14 days and stopped on DOL 20. Conventional mechanical ventilation was continued for five days. Clinical and radiological improvement was noted over the next week. Feedings resumed with EBM from DOL 15 and were tolerated well.

On DOL 29, two weeks after resuming enteral feeds with formula because of insufficient breast milk, the infant developed a second episode of bloody stools. The abdomen was soft, not distended, and non-tender, with 


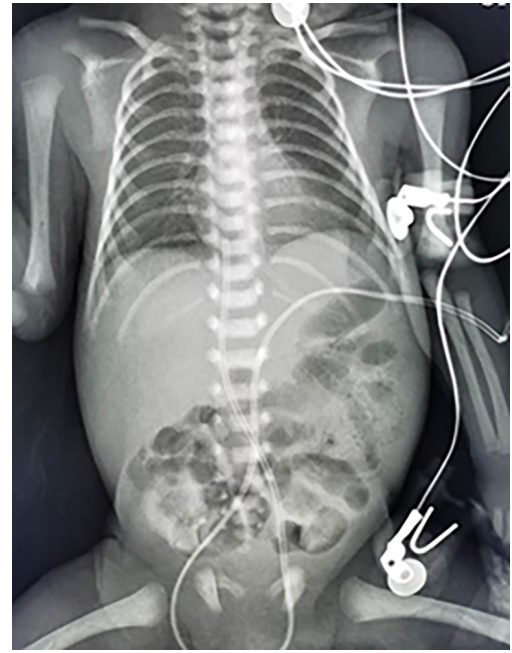

Figure 1 Abdominal radiograph demonstrated pneumatosis intestinalis (PI) of the descending colon on the day of life 7 with the first episode of bloody stools.

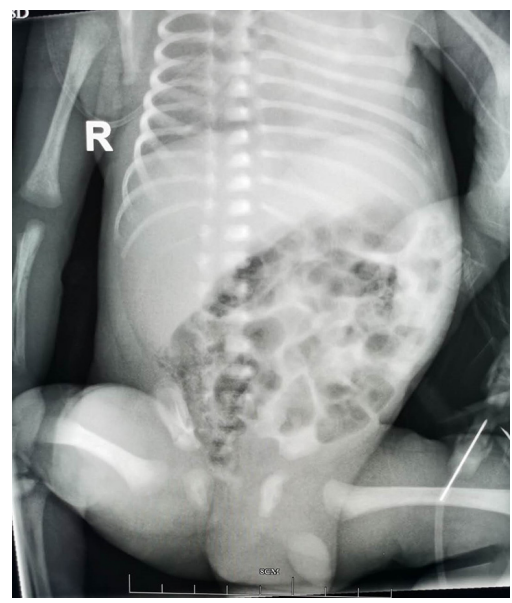

Figure 2 Abdominal radiograph revealed mild thickening of the small bowel with no evidence of pneumatosis intestinalis on the day of life 29 with the second episode of bloody stools.

active bowel sounds. The stool test was positive for occult blood, with normal CRP and WBC levels (Table 1). An abdominal radiograph revealed mild thickening of the small bowel, with no evidence of PI (Figure 2). Considering the reoccurrence of NEC, we started antibiotic therapy and suspended feeding again. Serial blood analysis showed normal CRP and WBC levels, so three days after discontinued feeding, enteral feeding with breast milk was gradually resumed. Antibiotics were suspended five days later with negative blood culture.
On DOL 46, the infant developed a third episode of bloody stool after feeding with expressed mother's milk $(140 \mathrm{~mL} / \mathrm{kg} / \mathrm{d})$ fortified with cow's milk. The infant was quite well, without emesis or abdominal distension, and the bowel sounds were found to be active. Laboratory tests showed decreased WBC levels, normal CRP levels, and a mild increase in eosinophil counts. With suspected FPIES, we suspended feeding, and the symptoms disappeared immediately. The infant continued to be fed expressed mother's milk fortified without cow's milk after one day of fasting, and after no bloody stools had occurred. We did not check IgE levels or stools tests for eosinophils because they were unavailable at our hospital.

On DOL 54, when preterm formula feeding was reinitiated during the outbreak of coronavirus disease 2019 (COVID-19), the infant developed abdominal distension with reocurrence of grossly bloody stools once again. Laboratory tests showed normal WBC and CRP levels. The eosinophil count showed a gradual increase over time (13.8\%) (Table 1). On suspicion of FPIES, the formula was changed to a hydrolyzed formula, resulting in a resolution of symptoms.

The 28-week premature infant was diagnosed with FPIES in addition to early-onset NEC. The recovery was complete with conservative management and hydrolyzed formula.

The patient was discharged home after 60 days of hospitalization, and a change to expressed mother's milk resulted in nonrecurrence of the symptoms. The infant was breastfed for 8 months after discharge, and no bloody stools recurred.

All procedures performed in studies involving human participants were in accordance with the ethical standards of the institutional and/or national research committee(s) and with the Helsinki Declaration (as revised in 2013). Written informed consent was obtained from the patient.

\section{Discussion}

There are unique aetiologies of bloody stools in neonates. Common causes of bloody stools in an otherwise healthy infant are anal fissures, swallowed maternal blood, vitamin $\mathrm{K}$ deficiency bleeding, and FPIES. When bloody stools occur in a premature infant in the neonatal intensive care unit (NICU), the possibility of NEC enters the differential diagnosis, although food allergy-induced enterocolitis can be causative and difficult to distinguish from NEC. At times, it may be difficult and important to differentiate the 
two conditions. However, there are very few study reports of preterm infants presenting with food allergy-induced enterocolitis after NEC. Here, we present a very-lowbirth-weight infant with recurrent episodes of bloody stools and increasing eosinophils who was finally diagnosed with FPIES after NEC recovery.

FPIES is a non-IgE-mediated food allergy that typically presents in infancy. The most common food allergy in infants is cow's milk allergy (CMA), which presents with a variety of gastrointestinal symptoms in the neonatal period, such as diarrhoea, vomiting, and bloody stool (9). Estimates of the true prevalence of FPIES vary greatly. A prospective birth cohort study noted a cumulative incidence of FPIES infants of 3 per 1,000 new-borns at a single hospital over 2 years $(0.34 \%)(10)$. A retrospective study observed that the incidence of cases with food-related symptoms that suggested CMA was $0.21 \%$ in neonatal care units in Japan (9). While an oral food challenge test for suspected CMA in infants tends to be avoided and limited wide-scale epidemiological information is available, the true prevalence rate for FPIES in infants is likely to be underestimated. Therefore, we must be aware that observations of infants with bloody stools that improve following the withdrawal of the suspected causal food confirm the diagnosis of FPIES.

FPIES is classified as a non- $\mathrm{IgE}$-mediated disorder that can involve antigen-specific $\mathrm{T}$ cells, antibodies, and cytokines as a cause of inflammation and is found in the colon by endoscopy and biopsy. However, the mechanistic link between NEC and FPIES is unknown. As a high incidence of CMA after gastroenterological surgery has been reported, we hypothesized a similar mechanism of CMA occurrence after gastroenterological inflammation (9). The sensitization mechanism may be associated with dysfunction of the gastrointestinal tract resulting from primary disease, excessive inflammation-induced intestinal mucosa damage, and atrophy in the intestinal mucosa caused by extended fasting after primary disease. This sensitization mechanism is assumed to cause increased intestinal mucosa permeability making the patient more vulnerable to CMA. According to this report, we suppose that premature infants are at risk for CMA and bovine-based fortifiers, especially after intestinal inflammation damage such as that caused by NEC.

In this case report, we confirmed an apparent increase in eosinophils in patients with FPIES occurred subsequent to NEC. Low-concentration, nonspecific eosinophilia is a frequent finding in preterm infants during hospitalization, and it has been described as blood eosinophilia in patients with infection, NEC, and packed red blood cell transfusion (11). Another report indicated that preterm infants with early persistent blood eosinophilia were at high risk during NEC convalescence (12). NEC is thought to be a major stimulus for prolonged eosinophil production, activation, and recruitment and adverse inflammatory outcomes during NEC. However, in this case, we observed a gradual increase in eosinophil counts after milk allergy exposure in NEC convalescence. This phenomenon may be linked to dysfunction of the gastrointestinal tract resulting from impaired intestinal mucosal and immunological defences or atrophy of the mucosa after NEC (13). The inflammatory process in FPIES may involve eosinophils. FPIES was associated with eosinophilic inflammation in histology of colonic biopsy in some cases (14). Infants with multiple food allergies have a high blood total eosinophil count (15). It is known that eosinophils can release cytokines and chemokines, that modulate inflammatory responses and may also affect tissue remodelling and fibrosis by releasing transforming growth factors (16). Thus, we suppose that infants have a higher incidence of blood eosinophilia and gastrointestinal food allergies after NEC.

FPIES is difficult to differentiate in the early stage of onset and is often misdiagnosed as NEC. Ultrasound findings suggest that PI, portal venous gas (PVG), bowel wall thickening, and weakened/absent intestinal motility can be found in both NEC and FPIES infants (17). Additionally, the radiological finding of PI is not specific to NEC (18). It is presumed that both NEC and FPIES cause intestinal mucosal injury in the involved intestines and cause PVG and PI. As a result, we considered recurrent NEC in the second episode of bloody stools, and we started with antibiotic therapy and suspended feeding. However, reviews of FPIES often show thrombocytosis and leucocytosis compared with leukopenia and thrombocytopenia in severe cases of NEC (7). In our case, the infant who developed FPIES appeared well without abdominal tenderness. Consecutive white blood cell count and CRP levels in the patient were normal. Abdominal ultrasound can also be used to differentiate FPIES from NEC in the early stage, with decreased or absent intestinal motility involving isolated segments of the bowel and $0 \%$ decreased intestinal motility in addition to the lesion segment. Although there was no abdominal ultrasound evaluation, our case suggests that infants presenting with bloody stools and normal abdominal $\mathrm{X}$-ray and laboratory tests are less likely to have NEC.

Oral food challenge is the gold standard for the diagnosis of food allergies. However, it is difficult to conduct this test 
to confirm food allergies in infants. History of an immediate allergic reaction and quick resolution of symptoms with food avoidance is often sufficient to establish a diagnosis of FPIES (19). The treatment of gastrointestinal food allergies requires the strict elimination of food allergens. A maternal elimination diet is often sufficient to controlling symptoms in breastfed infants, while in formula-fed infants, treatment usually involves the use of an extensively hydrolyzed or an amino acid-based formula (20). In this study, the patient's symptoms were quickly resolved by introducing a hydrolyzed formula. As such, complete resolution of signs and symptoms after elimination of cow's milk allergens further points to the diagnosis of FPIES.

\section{Conclusions}

Our case suggests that infants with recurrent episodes of bloody stools with increasing systemic eosinophils after NEC, should be considered for FPIES with cow's milk formula. Rapid improvement after removing exposure to milk protein and non-progression of systemic symptoms and signs may help differentiate FPIES from NEC.

\section{Acknowledgments}

Funding: This study was supported by Zhejiang Provincial Natural Science Foundation of China (Grant Number: 2021PY057, LY20H040008, LY15H040004).

\section{Footnote}

Reporting Checklist: The authors have completed the CARE reporting checklist. Available at http://dx.doi.org/10.21037/ tp-21-9

Peer Review File: Available at http://dx.doi.org/10.21037/tp21-9

Conflicts of Interest: Both authors have completed the ICMJE uniform disclosure form (available at http://dx.doi. org/10.21037/tp-21-9). The authors have no conflicts of interest to declare.

Ethical Statement: The authors are accountable for all aspects of the work in ensuring that questions related to the accuracy or integrity of any part of the work are appropriately investigated and resolved. All procedures performed in studies involving human participants were in accordance with the ethical standards of the institutional and national research committee and with the Helsinki Declaration (as revised in 2013). Written informed consent was obtained from the patient.

Open Access Statement: This is an Open Access article distributed in accordance with the Creative Commons Attribution-NonCommercial-NoDerivs 4.0 International License (CC BY-NC-ND 4.0), which permits the noncommercial replication and distribution of the article with the strict proviso that no changes or edits are made and the original work is properly cited (including links to both the formal publication through the relevant DOI and the license). See: https://creativecommons.org/licenses/by-nc-nd/4.0/.

\section{References}

1. Pai AK, Fox VL. Gastrointestinal Bleeding and Management. Pediatr Clin North Am 2017;64:543-61.

2. Raba AA, O'Sullivan A, Miletin J. Pathogenesis of necrotising enterocolitis: The impact of the altered gut microbiota and antibiotic exposure in preterm infants. Acta Paediatr 2021;110:433-40.

3. Moss RL, Kalish LA, Duggan C, et al. Clinical parameters do not adequately predict outcome in necrotizing enterocolitis: a multi-institutional study. J Perinatol 2008;28:665-74.

4. Mennini M, Fiocchi AG, Cafarotti A, et al. Food proteininduced allergic proctocolitis in infants: Literature review and proposal of a management protocol. World Allergy Organ J 2020;13:100471.

5. Martin VM, Virkud YV, Seay H, et al. Prospective Assessment of Pediatrician-Diagnosed Food ProteinInduced Allergic Proctocolitis by Gross or Occult Blood. J Allergy Clin Immunol Pract 2020;8:1692-9.e1.

6. Nowak-Węgrzyn A, Chehade M, Groetch ME, et al. International consensus guidelines for the diagnosis and management of food protein-induced enterocolitis syndrome: Executive summary-Workgroup Report of the Adverse Reactions to Foods Committee, American Academy of Allergy, Asthma \& Immunology. J Allergy Clin Immunol 2017;139:1111-26.e4.

7. Lenfestey MW, de la Cruz D, Neu J. Food ProteinInduced Enterocolitis Instead of Necrotizing Enterocolitis? A Neonatal Intensive Care Unit Case Series. J Pediatr 2018;200:270-3.

8. Srinivasan P, Brandler M, D'Souza A, et al. Allergic enterocolitis presenting as recurrent necrotizing enterocolitis 
in preterm neonates. J Perinatol 2010;30:431-3.

9. Miyazawa T, Itabashi K, Imai T. Retrospective multicenter survey on food-related symptoms suggestive of cow's milk allergy in NICU neonates. Allergol Int 2013;62:85-90.

10. Katz Y, Goldberg MR, Rajuan N, et al. The prevalence and natural course of food protein-induced enterocolitis syndrome to cow's milk: a large-scale, prospective population-based study. J Allergy Clin Immunol 2011;127:647-53.e1-3.

11. Juul SE, Haynes JW, McPherson RJ. Evaluation of eosinophilia in hospitalized preterm infants. J Perinatol 2005;25:182-8.

12. Wahidi LS, Sherman J, Miller MM, et al. Early Persistent Blood Eosinophilia in Necrotizing Enterocolitis Is a Predictor of Late Complications. Neonatology 2015;108:137-42.

13. Korai T, Kouchi K, Takenouchi A, et al. Neonates undergoing gastrointestinal surgery have a higher incidence of non-IgE-mediated gastrointestinal food allergies. Pediatr Surg Int 2018;34:1009-17.

14. Debuf MJ, Claeys T, Stalens JP, et al. Hematochezia

Cite this article as: Lu Y, Zhang ZQ. Food proteininduced enterocolitis syndrome presenting after necrotizing enterocolitis in a preterm neonate: a case report. Transl Pediatr 2021;10(5):1393-1398. doi: 10.21037/tp-21-9 caused by eosinophilic proctocolitis in a newborn before oral feeding: a case report. J Med Case Rep 2017;11:160.

15. Koksal BT, Barıs Z, Ozcay F, et al. Single and multiple food allergies in infants with proctocolitis. Allergol Immunopathol (Madr) 2018;46:3-8.

16. Kimura M, Shimomura M, Morishita H, et al. Eosinophilia in infants with food protein-induced enterocolitis syndrome in Japan. Allergol Int 2017;66:310-6.

17. Guo Y, Si S, Jia Z, et al. Differentiation of food proteininduced enterocolitis syndrome and necrotizing enterocolitis in neonates by abdominal sonography. J Pediatr (Rio J) 2021;97:219-24.

18. Liu H, Turner TWS. Allergic Colitis With Pneumatosis Intestinalis in an Infant. Pediatr Emerg Care 2018;34:e14-e15.

19. Feuille E, Nowak-Węgrzyn A. Food Protein-Induced Enterocolitis Syndrome, Allergic Proctocolitis, and Enteropathy. Curr Allergy Asthma Rep 2015;15:50.

20. Heine RG. Gastrointestinal food allergies. Chem Immunol Allergy 2015;101:171-80. 